\title{
Carpooling with ecologists, geographers and taxonomists: perceptions from conducting environmental research in tropical regions
}

\author{
Kate Baker ${ }^{1}$ D . Claas Damken ${ }^{2,5}$. Jean-Luc Gattolliat ${ }^{3}$. Ulmar Grafe ${ }^{4}$. \\ Rafhiah Kahar $^{5} \cdot$ Albert Orr $^{6} \cdot$ Michel Sartori $^{3} \cdot$ Rodzay A. Wahab $^{5} \cdot$ Herbert Zettel $^{7}$. \\ Michael A. Chadwick ${ }^{8}$
}

Received: 3 September 2018 / Revised: 5 December 2018 / Accepted: 23 December 2018 /

Published online: 18 January 2019

(C) The Author(s) 2019

\begin{abstract}
Greater than $80 \%$ of species on Earth are awaiting formal description, and simultaneously, many of these species unknown to science are becoming extinct. Here we highlight the importance and benefits of collaborating and working in interdisciplinary research groups, to improve quality and efficiency of both ecological and taxonomic research. The aim of this paper is to share and critique two methods used when conducting environmental field research in taxonomically data-poor parts of the world, such as Borneo. Through discussions with geographers, ecologists and taxonomists these two methods are evaluated. We conclude with a suggested solution to push taxonomic knowledge barriers by creating inter-disciplinary communities of researchers who work together to improve taxonomic identifications.
\end{abstract}

Keywords Insects $\cdot$ Interdisciplinary $\cdot$ Taxonomy $\cdot$ Citizen science $\cdot$ Field research . Tropical

\section{Introduction: what's the situation?}

It is suggested that over $80 \%$ of species on Earth are still awaiting formal description (Mora et al. 2011). The biodiversity knowledge gap, also known as the taxonomic impediment, varies geographically. Ecological theories underpinning our understanding of the biosphere stem mostly from well studied but relatively depauperate faunas and floras of temperate Europe, Russia and North America but have not been tested in the hyperdiverse tropics, thus hampering conservation and environmental management. The aim of this paper is to share and critique two research methods conducted in

Communicated by David Hawksworth.

\section{Kate Baker}

K.baker2@exeter.ac.uk

Extended author information available on the last page of the article 
a taxonomically understudied part of the world, the island of Borneo. The co-authors of this paper include geographers, ecologists and taxonomists.

The two approaches examined in this paper are examples of carpooling; a term coined by Halme et al. (2015) and used by Sheldon (2016), for collaborations between ecologists and taxonomists with mutual benefits to all. The first is the classic approach applied during a stream ecology research project which resulted in consulting communities including taxonomists, marine biologists, and professional underwater photographers. This first approach used a personal network of taxonomists via email to assist in the identifications. The second approach, titled the cascade identification method, was applied during an insect biodiversity survey which faced similar taxonomic challenges but approached the problems by using the citizen science portal iNaturlist.org, which maps and shares observations of global biodiversity.

\section{The classic approach: creating a personal network of taxonomists via Email}

In 2012, a research project commenced to investigate the basic eco-hydromorphic dynamics of pristine tropical stream systems in Ulu Temburong National Park (hereafter Belalong, one of the main rivers in the park) in Negara Brunei Darussalam, Borneo (Baker et al. 2016, 2017). The appeal of these remote tropical streams had a methodological downside, which was that little work had been conducted on the benthic macroinvertebrate communities. Despite ecological studies conducted over several years in the early 1990s by the Royal Geographical Society (with the Institute of British Geographers) and Universiti Brunei Darussalam (UBD), there were still significant amounts of baseline work required, especially on the streams and rivers.

The high number of undescribed benthic macroinvertebrates in the study streams of Belalong and lack of identification guides encouraged the first author to purchase an underwater camera to capture characteristics of the resident fauna (Fig. 1), and to attach a camera to a microscope for smaller macroinvertebrates. Initially, photography assisted in learning to identify and categorize the macroinvertebrates. After using the available keys to identify some of the animals, photography assisted in sharing the findings via email. At that time, there were no other researchers working on tropical macroinvertebrates at King's College London or at UBD. Therefore, to validate the identifications, the first author successfully contacted around 25 taxonomists specialised on tropical macroinvertebrates around the world.

These taxonomists were able to validate some identifications from the photos, and if it was not possible to identify from photographs specimens were sent by post. During the cause of the research project, personal visits were made to the Natural History Museums in London and Lausanne, where training on the latest taxonomic keys and specimen-preserving sampling techniques was received. This increased the use and value of the samples so that specimens could be used beyond the initial ecological research project and be stored in museum collections. These collaborations enabled the identification of macroinvertebrates more precisely than would been possible had the work been conducted solo. This increased the quality of the research outputs and has so far contributed to numerous first recordings for Brunei and Borneo and description of new taxa (e.g., Kaltenbach and Gattolliat 2017). 

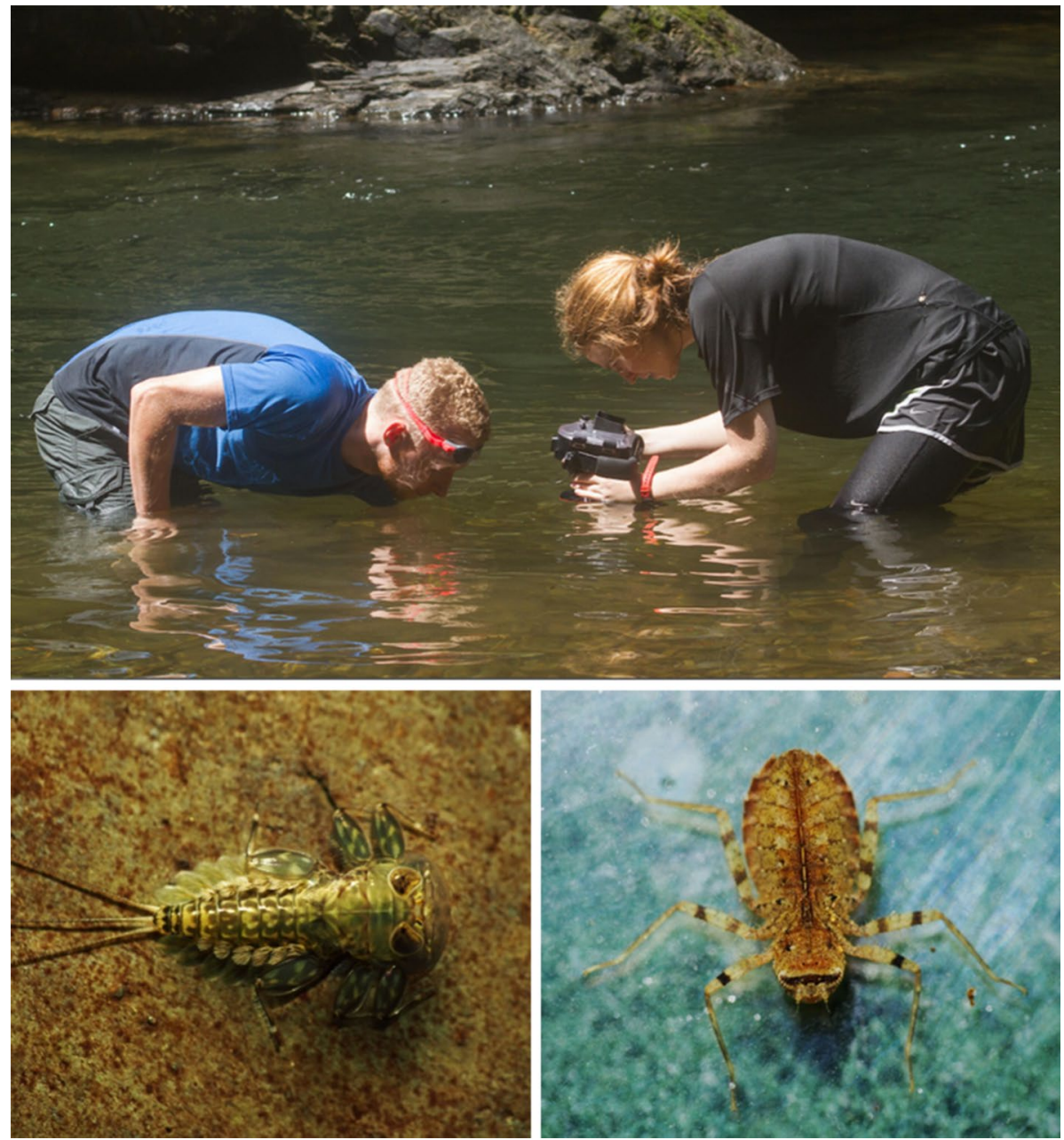

Fig. 1 Photography being conducted in Temburong National Park to capture characteristics of the resident fauna (top photo), Heptageniidae (flattened mayfly) (left photo) and a Macromia cydippe (Dragonfly) (right photo) Photo credit Top photo taken by Hanyrol Ahmad Sah and bottom two photos by Kate Baker

\section{The 'cascade' approach: crowd-sourcing identification using iNaturalist. org}

The 'network of taxonomists' method described above worked very well for the stream ecology research. However, it took many months to build up this network of taxonomists that specialise on tropical taxa. This was very useful for our research but not of direct use to other potential researcher groups. It is not possible to share this list as it would be a breach of data protection and trust, but it would be useful for other fellow researchers who are starting research in the tropics. It is not efficient for each researcher to have to start from scratch to create this type of network. Claas Damken, second author of this paper, a former Postdoctoral Research Fellow at the University of Brunei was investigating the diversity 
of heteropterans (true bugs) in the rainforests of the Sultanate around the same time the stream ecology project was conducted. He used the citizen science platform iNaturalist.org to interact with taxonomists to increase identification transparency and efficiency. Instead of communicating with taxonomists via email, the 'conversation' regarding the identification of the insects is conducted publicly on iNaturalist.org.

Citizen science platforms, such as iNaturlist.org, allow for uploading geo-referenced photos of species observations and species identifications by amateurs and professionals (Heberling and Isaac 2018). Although iNaturalist.org is designed to document observations of living specimens in their natural environment, it can also be used to handle professionally collected (i.e., under a collecting/research permit) and hence dead specimens. Importantly, geographic data of rare or endangered species can be obscured by the user. This 'cascade' identification method has three main steps:

Step one For ecologists working in species-rich areas or where up-to-date identification keys are lacking, the first step on the 'cascade' identification method is to sort samples to parataxonomic units (i.e., morphospecies or morphotypes) (Krell 2004) and to take high quality images of each 'species'. Then, add each 'species observation' to the website iNaturalist.org, and create a 'project' containing all the observations. Classify each species observation according to personal expertise to the lowest possible taxonomic rank. To increase the scientific value of these primary data, include environmental information such as sampling method or habitat. At some point during this first step, relevant taxonomists should be contacted and invited to join iNaturalist.org for the upcoming identification process.

Step two Sometimes a good photo is sufficient for a specialized taxonomist to identify conspicuous specimens to genus or even species rank. If it is not possible to get an ID based on a photo, the taxonomist can ask for further detail images or request a specimen for further physical examination. The ecologist should document on iNaturlist.org, who physically examined/identified a specimen in which year using which literature.

Step three The final and possibly most important step in the 'cascade' method is for the ecologists and taxonomists to commit to engage with new records of the target taxa in the target region made by other users of iNaturalist.org, be it during a different research project, a bioblitz or a stand-alone observation. This is made easy by either subscribing to a taxon in a specific region or simply saving a bookmark with relevant taxa/region already pre-selected in the search fields The ecologist/taxonomists will then receive notifications when people make observations of that specific taxon in that region and they can assist others users with their observations.

\section{Who benefits from the 'cascade' identification method?}

\section{The taxonomist}

Taxonomists are restricted by time and money (May 2010; Johnson 2012; Tahseen 2014). The 'cascade' identification method can potentially save time for the taxonomist by creating a space online, through the iNaturalist.org pages, that will host information on taxa which the ecologists can use to assist with morphotyping and/or identifying them with the community's help. Even in the tropics, common and conspicuous species often can be identified based on a good photograph. These specimens don't need to be sent to the taxonomist, thereby saving considerable time and money. Likewise, the taxonomist can comment on 
iNaturalist.org, if certain specimens belong to unidentifiable or difficult groups for which the taxonomist does not have the time/resources to identify. Again, not having to handle these specimens can save precious time for the taxonomist.

High resolution images of specimens identified by specialised taxonomists can be distributed beyond the iNaturalist portal, to increase the number of correctly identified images on the internet (for example through Google image search, Symbiota Collections of Arthropods Network Image Database, Wikipedia articles, Discover life database, etc.). Once good images of identified specimens of conspicuous species become more available online, taxonomists can save time for not having to identify the same species over and over again. This work can be done instead by the ecologists and other members of the wider community. Another advantage for both the taxonomist and the ecologist is that the taxonomist can become aware of interesting specimens in the early stages of a longer research project such as a $\mathrm{PhD}$ thesis. If the ecologist uploads images of specimens soon after collecting this can be relevant to the taxonomist if they are describing or revising taxa and additional specimens would be useful. Finally, iNaturalist organises and stores observations based on both geographic and taxonomic data. Using the map function, a taxonomist can easily search for potentially interesting observations at locations of interest, even if the particular observations are erroneously or not yet identified by the community (e.g., using the search term 'insects' to find hoverflies mistakenly identified as bees).

\section{The ecologist}

Ecologists benefit from the 'cascade' method as it creates a source and network of information to assist with identifications-including useful material and keys to assist with identification. The website helps to link ecologists to taxonomists, saving the ecologist time in searching for the taxonomists that specialise in certain regions. It also offers considerable savings in time for subsequent ecological studies, in nearby or similar habitats, as the next researcher can draw from the identifications made during and documented by the first study. Specimens identified to species rank by a professional can be 'tagged' with the species name, thus compiling distributional and phenological information for these species, which may be of use to ecological studies. At the time of submission of this manuscript, this cascade identification method proved to be an effective tool for the second author to handle more than 500 specimens of the large plant bug (Miridae) family, by collaborating with at least eight taxonomists. Finally, it brings transparency to the ecologist's identifications and research and allows future researchers to assess the quality of the identifications and thereby the data used in the original study (Krell 2004), which in turn enhances the scientific value of the original data.

\section{The wider community}

The wider community, can benefit from the 'cascade' method as it adds more specialist knowledge to the citizen science platform by increasing the specificity of the identification. It assists with knowledge exchange to the general public creating a link between the community and the 'scientists'. Studies that analyse species observations should acknowledge the source of the data; empowering the community to continue to contribute. Museums in the tropics need to be empowered and strengthened. This could be an important, possibly critical, part of the cascade method as the identified specimens can then deposited in countries of origin which are accessible to local researchers and thus 
provide incentives for them to get involved in taxonomic work. The location of museums and collections could be displayed on iNaturalist.org, encouraging the local community to engage in these centres.

The cascade approach does have its challenges which includes encouraging the different communities to upload images of specimens when they already know the ID and for them to stay engaged with the online tool to help other people with their IDs. Also, taking high resolution images is a time-consuming task, but will save time in the long run by building up taxonomic capacities.

\section{Conclusions}

In conclusion, we hope this paper highlights the importance and benefits of carpooling: collaborating and working in inter-disciplinary research groups. By collaborating using the 'cascade' method, it is possible to tackle the taxonomic impediment, use human resources more efficiently and push knowledge barriers to better understand how the ecosystem works. Researchers should look beyond their immediate colleagues and work in interdisciplinary projects including citizen science portals such as iNaturalist.org.

Acknowledgements Many thanks to Ken-ichi Ueda, co-founder of iNaturalist, and Peter Zwick for the engaging conversations and comments on this paper. We are grateful to the Universiti Brunei Darussalam, The International Consortium of Universities for the Study of Biodiversity and the Environment (iCUBE), as well as Brunei's Forestry Department and Brunei's Biodiversity Research and Innovation Centre. We extend a special thanks to the crew at Kuala Belalong Field Study Centre and Firdaus Ismail (undergraduate at UBD). Kate Baker was funded by a Natural Environment Research Council Ph.D. studentship (code: DAR7132). Claas Damken was funded by a Universiti Brunei Darussalam Postdoctoral Fellowship.

Author contributions KB: Writing the manuscript, conceiving the idea of the study and leading the discussion through email and conversations. CD, MC: Writing the manuscript; conceiving the idea of the study-through email and conversations. MS, J-LG, HZ, AO: conceiving the idea of the study-through email and conversations; writing/editing text. UG, RAW, RK: conceiving the idea of the study- through email and conversations; writing/editing text; involved in the process of field research in Borneo.

Open Access This article is distributed under the terms of the Creative Commons Attribution 4.0 International License (http://creativecommons.org/licenses/by/4.0/), which permits unrestricted use, distribution, and reproduction in any medium, provided you give appropriate credit to the original author(s) and the source, provide a link to the Creative Commons license, and indicate if changes were made.

\section{References}

Baker K, Chadwick MA, Kahar R, Sulaiman Z, Wahab RA (2016) Fluvial biotopes influence macroinvertebrate biodiversity in South-East Asian tropical streams. Ecosphere 7:12. https://doi. org/10.1002/ecs2.1479

Baker K, Chadwick MA, Wahab RA, Kahar R (2017) Benthic community structure and ecosystem functions in above- and below-waterfall pools in Borneo. Hydrobiologia 787(1):1-16. https://doi. org/10.1007/s10750-016-2975-4

Halme P, Kuusela S, Juslén A (2015) Why taxonomists and ecologists are not, but should be, carpooling? Biodivers Conserv 24:1831-1836. https://doi.org/10.1007/s10531-015-0899-3

Heberling JM, Isaac BL (2018) iNaturalist as a tool to expand the research value of museum specimens. Appl Plant Sci. https://doi.org/10.1002/aps3.1193 
Johnson NF (2012) A collaborative, integrated and electronic future for taxonomy. Invertebr Syst 25(5):471-475. https://doi.org/10.1071/IS11052

Kaltenbach T, Gattolliat JL (2017) New species of Indocloeon Müller-Liebenau from South-East Asia (Ephemeroptera, Baetidae). ZooKeys 723:43-60. https://doi.org/10.3897/zookeys.723.20578

Krell FT (2004) Parataxonomy vs. taxonomy in biodiversity studies-pitfalls and applicability of 'morphospecies' sorting. Biodivers Conserv 13(4):795-812. https://doi.org/10.1023/b:bioc.0000011727 .53780 .63

May RM (2010) Tropical arthropod species, more or less? Science 329(5987):41-42. https://doi. org/10.1126/science. 1191058

Mora C, Tittensor DP, Adl S, Simpson AG, Worm B (2011) How many species are there on Earth and in the ocean? PLoS Biol 9:8. https://doi.org/10.1371/journal.pbio.1001127

Sheldon AL (2016) Mutualism (carpooling) of ecologists and taxonomists. Biodivers Conserv 25:187-191. https://doi.org/10.1007/s10531-015-1032-3

Tahseen Q (2014) Taxonomy: the crucial yet misunderstood and disregarded tool for studying biodiversity. J Biodiv Endanger Species 2:3. https://doi.org/10.4172/2332-2543.1000128

\section{Affiliations}

\section{Kate Baker ${ }^{1}\left[\right.$. Claas Damken ${ }^{2,5}$. Jean-Luc Gattolliat ${ }^{3}$. Ulmar Grafe ${ }^{4}$. Rafhiah Kahar ${ }^{5} \cdot$ Albert Orr $^{6} \cdot$ Michel Sartori $^{3} \cdot$ Rodzay A. Wahab ${ }^{5} \cdot$ Herbert Zettel $^{7}$. Michael A. Chadwick ${ }^{8}$}

1 Centre for Water Systems, College of Engineering, Mathematics and Physical Sciences, University of Exeter, North Park Road, Harrison Building, Exeter EX4 4QF, UK

2150 Portobello Road, Dunedin 9013, New Zealand

3 Department of Ecology and Evolution, Biophore, University of Lausanne, 1015 Lausanne, Switzerland

4 Department of Biology, Universiti Brunei Darussalam, Jalan Universiti, Bandar Seri Begawan BE1410, Brunei Darussalam

5 Institute for Biodiversity and Environmental Research, Universiti Brunei Darussalam, Jalan Universiti, Bandar Seri Begawan BE1410, Brunei Darussalam

6 Environmental Futures Research Institute, Griffith University, Nathan, QLD 4111, Australia

7 Natural History Museum, International Research Institute for Entomology, Burgring 7, 1010 Vienna, Austria

8 Department of Geography, King's College London, Bush House (NE) 5.22, London WC2B 4BG, UK 